http://dx.doi.org/10.1590/1678-4162-8605

Arq. Bras. Med. Vet. Zootec., v.69, n.1, p.123-129, 2017

\title{
Produção e caracterização de protease fibrinolítica de Streptomyces parvulus DPUA 1573
}

\author{
[Production and characterization of fibrinolytic protease from \\ Streptomyces parvulus DPUA 1573] \\ J.M.S. Batista ${ }^{1}$, E.L. Clementino ${ }^{1}$, T.P. Nascimento ${ }^{2}$, G.M.S. Lima $^{2}$, T.S. Porto ${ }^{3}$, \\ A.L.F. Porto ${ }^{1 *}$ C.S. Porto ${ }^{4}$ \\ ${ }^{1}$ Universidade Federal Rural de Pernambuco - Recife, PE \\ ${ }^{2}$ Universidade Federal de Pernambuco - Recife, PE \\ ${ }^{3}$ Unidade Acadêmica de Garanhuns, UFRPE - Garanhuns, PE \\ ${ }^{4}$ Universidade Federal de Alagoas, UFAL - Penedo, AL
}

\begin{abstract}
RESUMO
As proteases fibrinolíticas são capazes de degradar coágulos de fibrina formados dentro dos vasos sanguíneos, evitando a trombose intravascular. Em animais, a tromboflebite, que acomete frequentemente os equinos, ocasiona, em seus casos graves, a obstrução jugular e também um edema de laringe, derivando a obstrução das vias aéreas, o que possibilita um edema cerebral, ocorrendo o óbito do animal. Devido ao fato de o tratamento ser de custo elevado, faz-se necessária a investigação de outras fontesde proteases fibrinolíticas com custos menores e com menos efeitos colaterais. Diante disso, este estudo tem como objetivo produzir e caracterizar proteases fibrinolíticas obtidas de Streptomyces parvulus DPUA 1573. Para produção da enzima, foi utilizado um planejamento fatorial $2^{4}$ avaliando a concentração da farinha de soja $(0,5,1,0$ e $1,5 \%)$ e da glicose $(0,0,5$ e $1,0 \mathrm{~g} / \mathrm{L})$, temperatura $\left(28,32\right.$ e $\left.37^{\circ} \mathrm{C}\right)$ e agitação $(150,200$ e $250 \mathrm{rpm})$ sobre a biomassa e a atividade fibrinolítica. Pode-se verificar que a protease fibrinolítica apresentou atividade máxima $(835 \mathrm{U} / \mathrm{mL})$ nas condições de concentração de $1,5 \%$ de soja, $1 \mathrm{~g} / \mathrm{L}$ de glicose, $28^{\circ} \mathrm{C}$ e $150 \mathrm{rpm}$ com 48 horas de fermentação. A protease fibrinolítica obtida teve temperatura e $\mathrm{pH}$ ótimos de $55^{\circ} \mathrm{C}$ e $\mathrm{pH} 9,0$, respectivamente. A atividade enzimática foi inibida pelo EDTA, pelo íon $\mathrm{Fe}^{2+}$ e pelo SDS, o que indicou a enzima ser uma metaloprotease. A linhagem Streptomyces parvulus DPUA 1573 foi capaz de produzir protease fibrinolítica, possuindo características bioquímicas favoráveis à aplicação na medicina veterinária e possivelmente humana.
\end{abstract}

Palavras-chave: Streptomyces parvulus, proteases fibrinolíticas, fermentação submersa

\begin{abstract}
Fibrinolytic proteases are able to degrade fibrin clot formed in the blood vessel, avoiding intravascular thrombosis. In animals, thrombophlebitis often affects horses, and in severe cases causes obstruction of the jugular and laryngeal edema leading to airway obstruction allowing cerebral edema resulting in the death of the animal. Since treatment is costly, the investigation of other sources of fibrinolytic proteases at lower cost and with fewer side effects is needed. Thus, this study aims to produce and characterize fibrinolytic proteases from Streptomyces parvulus DPUA 1573. For enzyme production, a factorial design was performed to evaluate $2^{4}$ soybean flour concentration $(0.5,1.0$ and $1.5 \%)$ and glucose $(0,0.5$ and $1.0 \mathrm{~g} / \mathrm{L})$, temperature $(28,32$ and $\left.37^{\circ} \mathrm{C}\right)$ and agitation $(150,200$ and $250 \mathrm{rpm})$ on biomass and fibrinolytic activity. Fibrinolytic protease showed maximum activity $(835 \mathrm{U} / \mathrm{mL})$ under these conditions: $1.5 \%$ soybean flour, $1 \mathrm{~g} / \mathrm{L}$ glucose, $28^{\circ} \mathrm{C}$, and $150 \mathrm{rpm} 48$ hours of fermentation. The optimal temperature was $55^{\circ} \mathrm{C}$ and optimal $\mathrm{pH}$ was 9.0. Fibrinolytic protease activity was inhibited by EDTA, the ion $\mathrm{Fe}^{2+}$, and by SDS, which indicated that the enzyme is a metalloprotease. The strain Streptomyces parvulus DPUA 1573 was able to produce fibrinolytic protease with biochemical characteristics favorable for application in veterinary and human medicine.
\end{abstract}

Keywords: Streptomyces parvulus, fibrinolytic protease, submerged fermentation

Recebido em 22 de julho de 2015

Aceito em 9 de agosto de 2016

*Autor para correspondência (corresponding author)

E-mail: analuporto@yahoo.com.br 


\section{INTRODUÇÃO}

As proteases são importantes, pois atuam no metabolismo e na regulação metabólica, podendo ser encontradas em diferentes organismos, e seu mecanismo envolve a capacidade de hidrolisar ligações peptídicas (Castro et al., 2011). Entre as proteases, destacam-se as com potencial fibrinolítico, que são enzimas com capacidade de degradar a fibrina, proteína de maior componente dos coágulos sanguíneos. A formação do coágulo de fibrina é normalmente regulada pelo sistema biológico. Entretanto, quando a fibrina não é hidrolisada, ocorre a trombose, bem como outros distúrbios cardiovasculares (Choi et al., 2011; Nascimento et al., 2016).

Os distúrbios cardiovasculares também são relevantes na medicina veterinária. Os equinos são afetados por processos trombolíticos, entre estes se destacam a trombose aortoilíaca e a tromboflebite (Oyamada et al., 2007; Sobreira et al., 2008; Escodro et al., 2010). A tromboflebite é ocasionada pelo uso prolongado de cateteres venosos ou de injeções intravenosas que causam lesão mecânica ou química na parede do vaso, resultando em oclusão completa da veia. Para o tratamento dessa doença, a medicina veterinária utiliza antitrombóticos, antiagregantes plaquetários e anti-inflamatórios. No entanto, há uma carência em medicamentos mais acessíveis, com baixo custo, e também de tratamentos mais eficazes (Barbosa et al., 2009). Em humanos, o tratamento é realizado com agentes trombolíticos, tais como t-PA (ativador plasminogênio tecidual) e uroquinase (Cicha, 2015). Considerando-se a necessidade de buscar novos agentes fibrinolíticos seguros e de baixo custo e de evitar cirurgia no caso dos equinos, estudos estão sendo realizados sobre novas proteases fibrinolíticas.

As bactérias são utilizadas biotecnologicamente, destacando as actinobactérias do gênero Streptomyces, devido a sua capacidade de produzir enzimas extracelulares, como proteases fibrinolíticas (Silva et al., 2016) e biocompostos com variadas atividades biológicas, como antibacterianos e antiparasitários (Demir et al., 2015; Balachandran et al., 2016). Este trabalho teve como objetivo produzir e caracterizar proteases fibrinolíticas obtidas de Streptomyces parvulus DPUA 1573.

\section{MATERIAL E MÉTODOS}

A linhagem de Streptomyces parvulus DPUA 1573 foi isolada de líquens da Região Amazônica e pertence à coleção de culturas DPUA (Departamento de Parasitologia da Universidade Federal do Amazonas), gentilmente cedida pela prof. ${ }^{a}$ Maria Francisca Simas Teixeira. O micro-organismo isolado foi mantido no meio ISP-2 (Pridham et al., 1957), com glicerol $10 \%, \mathrm{a}-20^{\circ} \mathrm{C}$.

Para a produção de protease fibrinolítica, foi utilizado o meio MS-2 (Porto et al., 1996), pH 7 autoclavado a $121^{\circ} \mathrm{C} / 1 \mathrm{~atm}$, por 20 minutos. Foram utilizados Erlenmeyers $(125 \mathrm{~mL})$ contendo $50 \mathrm{~mL}$ de meio de cultura, sendo o inóculo correspondente a $10^{8} \mathrm{UFC} / \mathrm{mL}$. O crescimento celular, a curva de $\mathrm{pH}$, a determinação da atividade fibrinolítica e a atividade proteásica foram determinados a cada 24 horas até 120 horas de fermentação. Para determinar a melhor condição para produção da protease fibrinolítica, utilizou-se um planejamento fatorial $2^{4}$, conforme a Tab. 1, considerando-se como variáveis independentes a concentração da fonte de nitrogênio (soja), a concentração da fonte de carbono (glicose), a temperatura e a agitação. Consideraram-se como variáveis respostas os valores da biomassa e a atividade fibrinolítica. As condições estudadas, bem como possíveis interações, foram analisadas estatisticamente com o auxílio do programa Statistica 8.0 (Statistica, 2008) com nível de significância de $95 \%$.

Tabela 1. Níveis das variáveis do planejamento fatorial $2^{4}$ para produção de protease fibrinolítica por Streptomyces parvulus DPUA 1573

\begin{tabular}{lccc} 
& \multicolumn{3}{c}{ Níveis } \\
\cline { 2 - 4 } \multicolumn{1}{c}{ Variáveis } & Menor $(-1)$ & Central $(0)$ & Maior $(+1)$ \\
\hline Concentração do filtrado de soja (\%) & 0,5 & 1 & 1,5 \\
Concentração de glicose (g/L) & 0 & 0,5 & 1 \\
Agitação (rpm) & 150 & 200 & 250 \\
Temperatura $\left({ }^{\circ} \mathrm{C}\right)$ & 28 & 32 & 37 \\
\hline
\end{tabular}


A determinação da atividade proteásica foi realizada pelo método de Ginther (1979), e a atividade fibrinolítica foi realizada de acordo com Wang et al. (2011). Na caracterização enzimática, foram avaliados o efeito e a estabilidade do $\mathrm{pH}$ e da temperatura, os íons metálicos, surfactantes e os inibidores sobre a atividade proteásica. Para estudo do efeito ao $\mathrm{pH}$, o extrato enzimático foi incubado a diferentes tampões: citrato-fosfato ( $\mathrm{pH} 4,0$ a 6,0), fosfato de sódio ( $\mathrm{pH}$ 6,0 e 7,0), Tris- $\mathrm{HCl}(\mathrm{pH} \mathrm{7,0,} \mathrm{8,0} \mathrm{e}$ 9,0), carbonato-bicarbonato $(\mathrm{pH} 9,0$ e 10,0) a $37^{\circ} \mathrm{C}$ por uma hora. $\mathrm{O}$ efeito da temperatura foi determinado pela incubação do extrato enzimático em temperaturas variando entre $10^{\circ} \mathrm{C}$ e $100^{\circ} \mathrm{C}$, por um período de uma hora. Para a estabilidade à temperatura, a incubação ocorreu durante três horas, sendo sua atividade enzimática determinada a cada 30 minutos. Em relação ao efeito dos íons e de inibidores, estes foram escolhidos e realizados de acordo com Nascimento et al. (2016).

Os surfactantes testados foram SDS (dodecilsulfato de sódio $-\mathrm{C}_{12} \mathrm{H}_{25} \mathrm{SO}_{4} \mathrm{Na}$ ), Triton $\mathrm{X}-100 \quad\left(\mathrm{C}_{14} \mathrm{H}_{22} \mathrm{O}\left(\mathrm{C}_{2} \mathrm{H}_{4} \mathrm{O}\right)_{n}\right)$, Tween 20 (monopalmitato de polioxietileno sorbitano $\mathrm{C}_{58} \mathrm{H}_{114} \mathrm{O}_{26}$ ) e Tween 80 (monopalmitato de polioxietileno sorbitano $-\mathrm{C}_{64} \mathrm{H}_{124} \mathrm{O}_{26}$ ), estudados nas seguintes concentrações: $0,5 \%$; $1,0 \%, 1,5 \%$ e $2,0 \%$.

\section{RESULTADOS E DISCUSSÃO}

O crescimento da linhagem Streptomyces parvulus DPUA 1573 (Fig. 1) não apresentou fase lag, uma vez que a concentração do inóculo facilitou sua adaptação ao meio de produção. A fase logarítmica ocorreu $48 \mathrm{~h}$ de fermentação, a fase estacionária em que o crescimento é desacelerado (96h), devido à diminuição de nutrientes no meio. A fase de declínio foi observada a partir de 120h, todos esses fenômenos foram semelhantes ao estudo de Narayana e Vijayalakshmi (2008), no qual descreve-se a produção da enzima amilase pelo Streptomyces albidoflavus com a produção máxima de biomassa com 96 horas.

Durante o processo de fermentação, o $\mathrm{pH}$ variou de 7,0 (0h) para 9,0 (120h). Essa alteração pode ser resultante da formação de amônia no meio fermentativo, possivelmente pela desaminação das proteínas existentes na fonte de nitrogênio (farinha de soja). Silva et al. (2016), ao estudarem a produção de protease fibrinolítica por Streptomyces sp. DPUA 1576 utilizando o mesmo meio de produção, observaram que o aumento do $\mathrm{pH}$ favorece a produção enzimática.

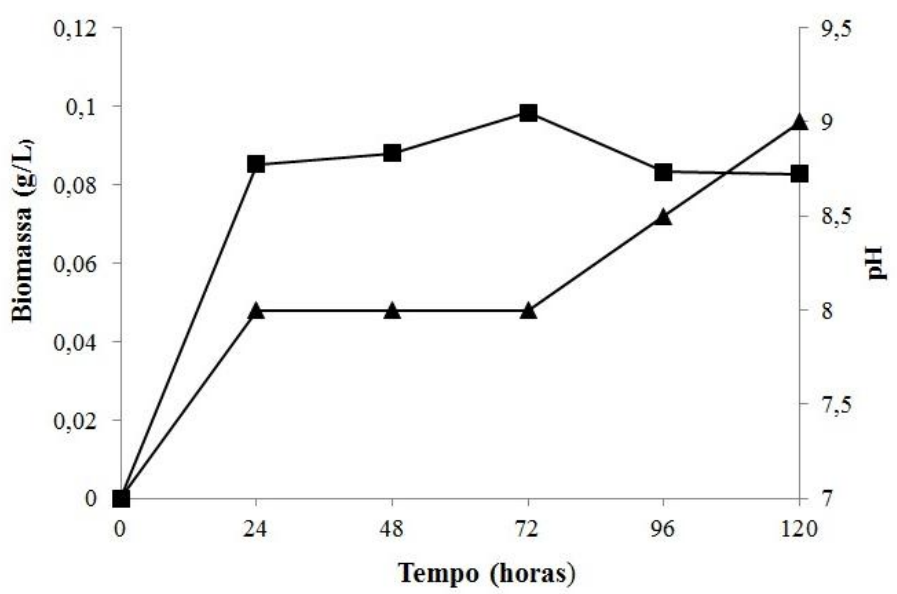

Figura 1. Curvas de $\mathrm{pH}(\boldsymbol{\Delta})$ e crescimento do micro-organismo(a) Streptomyces parvulus DPUA 1573 no meio MS-2.

No presente estudo, verificaram-se $48 \mathrm{~h}$ como o melhor tempo de produção da enzima. Esse curto tempo se torna atrativo para a indústria pelos custos menores na produção da enzima de interesse. O contrário foi visto por Silva et al. (2016), os quais, ao estudarem o tempo de produção da protease fibrinolítica por Streptomyces sp. DPUA 1576, observaram que, a partir de $72 \mathrm{~h}$, foram obtidos maiores valores em atividade fibrinolítica. 
Os valores de atividade fibrinolítica no planejamento fatorial variaram de 625,0 a 835,0 $\mathrm{U} / \mathrm{mL}$ (Tab. 2). A condição que apresentou maior valor em atividade fibrinolítica $(835,0 \mathrm{U} / \mathrm{mL})$ com 48 horas de fermentação foi com $1,5 \%$ de farina de soja, $1 \mathrm{~g} / \mathrm{L}$ de glicose, $28^{\circ} \mathrm{C}$ e $150 \mathrm{rpm}$. Esses resultados de atividade fibrinolítica foram superiores aos apresentados por Streptomyces venezuelae (16U/mL) (Bhavani et al., 2012).

Tabela 2. Resultados de atividade fibrinolítica obtidos no planejamento fatorial $2^{4}$ com 48 horas de fermentação produzidas pelo Streptomyces parvulus DPUA 1573.

\begin{tabular}{cccccc}
\hline Ensaios & $\begin{array}{c}\text { Soja } \\
(\%)\end{array}$ & $\begin{array}{c}\text { Glicose } \\
(\mathrm{g} / \mathrm{L})\end{array}$ & $\begin{array}{c}\text { Temperatura } \\
\left({ }^{\circ} \mathrm{C}\right)\end{array}$ & $\begin{array}{c}\text { Agitação } \\
(\mathrm{rpm})\end{array}$ & $\begin{array}{c}\text { Atividade fibrinolítica } \\
(\mathrm{U} / \mathrm{mL})\end{array}$ \\
\hline 1 & 0,5 & 0 & 28 & 150 & 632,5 \\
2 & 1,5 & 0 & 28 & 150 & 655,0 \\
3 & 0,5 & 1 & 28 & 150 & 752,5 \\
4 & 1,5 & 1 & 28 & 150 & 835,0 \\
5 & 0,5 & 0 & 37 & 150 & 822,5 \\
6 & 1,5 & 0 & 37 & 150 & 662,5 \\
7 & 0,5 & 1 & 37 & 150 & 667,5 \\
8 & 1,5 & 1 & 37 & 150 & 702,5 \\
9 & 0,5 & 0 & 28 & 250 & 677,5 \\
10 & 1,5 & 0 & 28 & 250 & 732,5 \\
11 & 0,5 & 1 & 28 & 250 & 625,0 \\
12 & 1,5 & 1 & 28 & 250 & 772,5 \\
13 & 0,5 & 0 & 37 & 250 & 692,5 \\
14 & 1,5 & 0 & 37 & 250 & 685,0 \\
15 & 0,5 & 1 & 37 & 250 & 727,5 \\
16 & 1,5 & 1 & 37 & 250 & 747,5 \\
$17 \mathrm{C}$ & 1,0 & 0,5 & 32 & 200 & 680,0 \\
$18 \mathrm{C}$ & 1,0 & 0,5 & 32 & 200 & 672,5 \\
$19 \mathrm{C}$ & 1,0 & 0,5 & 32 & 200 & \\
$20 \mathrm{C}$ & 1,0 & 0,5 & 32 & & 65,0 \\
\hline
\end{tabular}

A variável independente que isoladamente teve significância foi a concentração de glicose, a qual influenciou positivamente a produção da protease fibrinolítica (Fig. 2A). Sanchez e Demain (2002) afirmaram que as fontes de carbono, como a glicose, são comumente utilizadas para favorecer o crescimento do microorganismo, melhorando, assim, a produção de enzimas e metabólitos secundários. Por outro lado, Silva et al. (2015), ao estudarem a produção de protease fibrinolítica por Streptomyces sp. DPUA 1576, descreveram que apenas a fonte de nitrogênio (soja) foi significativa.
A interação das variáveis independentes, concentração da fonte de carbono (glicose), temperatura e agitação, apresentou um efeito significativo e positivo (Fig. 3A), indicando que as melhores condições de produção da enzima fibrinolítica foram encontradas em $1 \%$ de glicose, $28^{\circ} \mathrm{C}$ e $150 \mathrm{rpm}$ (Fig. 3B). O estudo realizado por Bhavani et al. (2012) com a linhagem mutante Streptomyces venezuelae obteve melhor resultado na produção da protease fibrinolítica a $40^{\circ} \mathrm{C}$, apresentando atividade de 16 $\mathrm{U} / \mathrm{mL}$, enquanto neste trabalho a temperatura selecionada foi de $28^{\circ} \mathrm{C}$. 

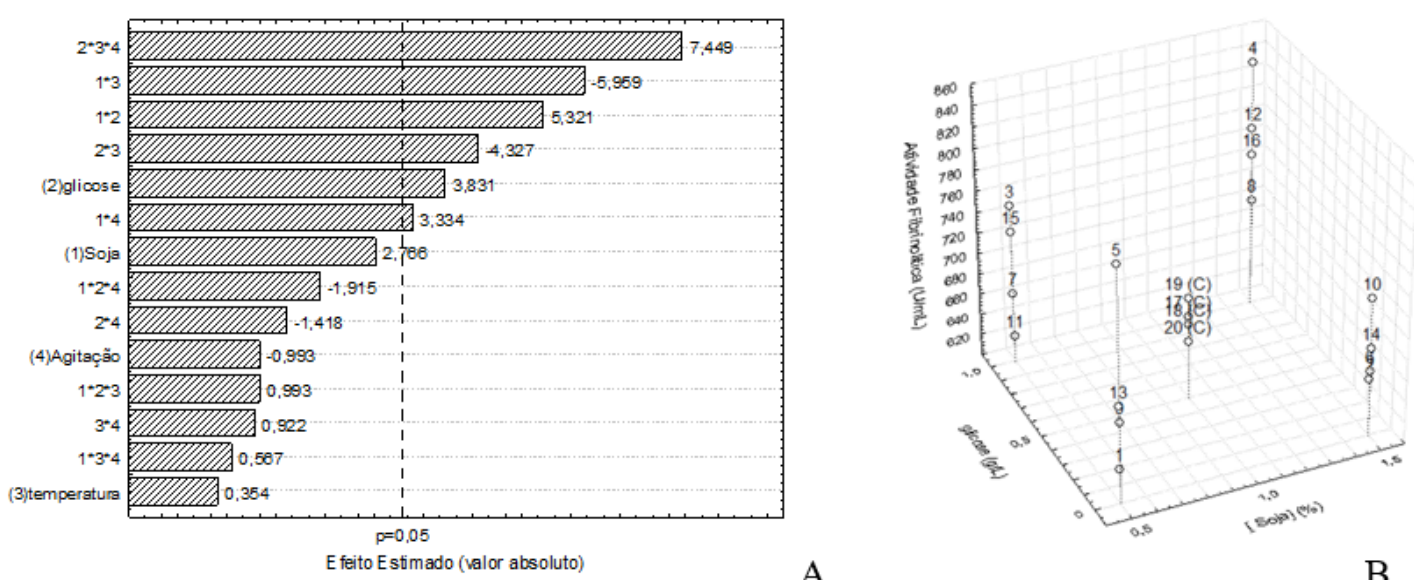

A

Figura 2. A - Gráfico de Pareto na produção de protease fibrinolítica por Streptomyces parvulus DPUA 1573. B - Representação gráfica tridimensional da relação entre a concentração de soja e de glicose na produção de protease fibrinolítica por Streptomyces parvulus DPUA 1573 no tempo de 48h.

$\mathrm{Na}$ caracterização da protease, a temperatura ótima foi de $55^{\circ} \mathrm{C}$ (Fig.3A). Entretanto, observase que a atividade da enzima se manteve estável, com temperaturas até $40^{\circ} \mathrm{C}$, sendo conservadas $100 \%$ de sua atividade por um período de três horas. Esses dados são semelhantes aos resultados obtidos por $\mathrm{Ju}$ et al.(2012), que estudaram a enzima fibrinolítica produzida pelo Streptomyces sp., a qual se manteve estável também a $30^{\circ} \mathrm{C}$.

Em relação ao efeito do $\mathrm{pH}$, a protease em estudo se manteve estável em valores de $\mathrm{pH}$ entre 7,0 e 9,0 (Fig. 3B). Adicionalmente, foi observado que no $\mathrm{pH}$ 9,0 a atividade proteásica foi máxima, caracterizando o $\mathrm{pH}$ ótimo. Simkhada et al. (2010) afirmam que a faixa de $\mathrm{pH}$ neutro ou alcalino apresentou melhores resultados para a produção de uma protease fibrinolítica produzida pelo Streptomyces sp. CS684; além disso, as proteases alcalinas obtidas por micro-organismos têm atraído grande atenção das indústrias devido a sua elevada atividade catalítica e ao alto grau de especificidade ao substrato.
Nos ensaios de inibição, ocorreu redução da atividade da protease com presença de EDTA $(31,84 \%)$ e $\beta$-mercaptoetanol (2,02\%) (Tab. 3). Os resultados indicaram que a protease estudada pertence à classe das metaloproteases. As proteases fibrinolíticas que pertencem a esta classe exigem metal bivalente para suas atividades, de modo que estas podem ser inibidas por agentes quelantes, como o EDTA (Mander $e t$ al., 2011). Nos estudos de caracterização realizados por $\mathrm{Ju}$ et al. (2012), o EDTA foi o principal agente inibidor. Mander et al. (2011) também verificaram a ação inibitória do EDTA, classificando a enzima como metaloprotease.

Diante dos íons estudados, foi observado que o íon $\mathrm{Ca}^{2+}$ nas concentrações de 10 e $20 \mathrm{mM}$ obteve a ativação superior a $22 \%$, porém não sofreu inibição pelo íon K. Entretanto, a atividade proteásica sofreu maior inibição $(42,3 \%)$ pelo íon $\mathrm{Zn}^{2+}$, corroborando os resultados obtidos por Simkhada et al. (2010), que também tiveram a mesma inibição para a protease fibrinolítica produzida pelo Streptomyces sp. CS684. 


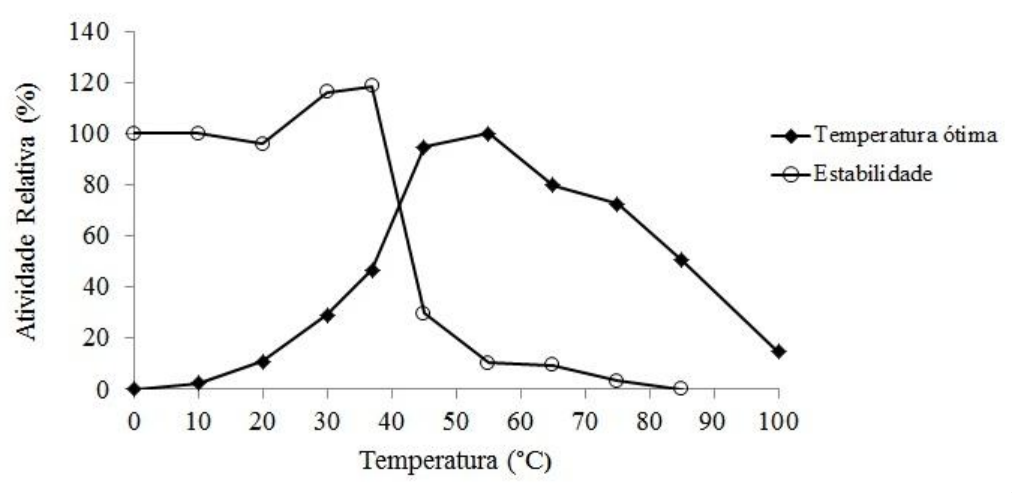

A

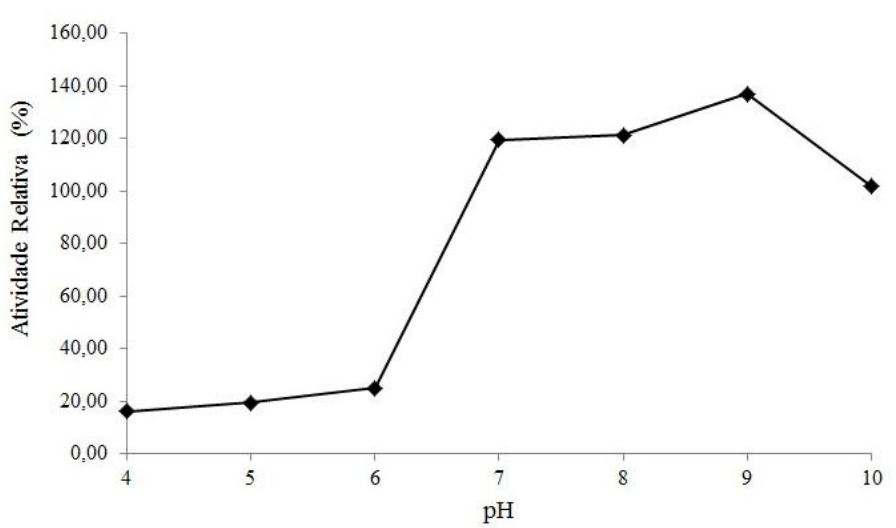

Figura 3. A - Temperatura ótima e estabilidade da protease fibrinolítica. B - Curva do pH ótimo da protease obtida por Streptomyces parvulus DPUA 1573 no tempo de $48 \mathrm{~h}$.

Tabela 3. Efeito de inibidores na atividade de proteases produzida por Streptomyces parvulus DPUA 1573

\begin{tabular}{cc}
\hline Inibidores & $\begin{array}{c}\text { Atividade proteásica } \\
\text { residual }(\%)\end{array}$ \\
\hline Controle & 100,0 \\
EDTA & 68,1 \\
PMSF & 168,2 \\
Pepstatina A & 121,4 \\
$\beta$-mercaptoetanol & 97,9 \\
Ácido iodoacético & 189,5 \\
\hline
\end{tabular}

Dos surfactantes avaliados, a atividade da protease foi ativada pelo Tween $80(11,33 \%)$ e principalmente pelo SDS $(51,78 \%)$, ambos com concentração de $2 \%$. Esses resultados são semelhantes ao do estudo de Wang et al. (2011), pelo qual o SDS teve um efeito estimulador sobre a atividade da enzima fibrinolítica produzida por Bacillus subtilis TKU007, podendo ser justificado pela alteração na conformação da enzima, aumentando, assim, a sua acessibilidade com o substrato.

\section{CONCLUSÕES}

A linhagem Streptomyces parvulus DPUA 1573 foi capaz de produzir protease fibrinolítica em todas as condições de fermentação. No entanto, a protease fibrinolítica apresenta atividade máxima $(835 \mathrm{U} / \mathrm{mL})$ nas condições de concentração de $1,5 \%$ de soja, $1 \mathrm{~g} / \mathrm{L}$ de glicose, $28^{\circ} \mathrm{C}$ e $150 \mathrm{rpm}$ com 48 horas de fermentação. A protease produzida pelo Streptomyces parvulus DPUA 1573 é estável na faixa de $\mathrm{pH}$ entre 7 e 9 , apresentando propriedades interessantes para a indústria farmacêutica, visando a possíveis aplicações na área de medicina veterinária. 


\section{REFERÊNCIAS}

BALACHANDRAN, C.; DURAIPANDIYAN, V.; ARUN, Y. et al. Isolation and characterization of 2hydroxy-9,10-anthraquinone from Streptomyces olivochromogenes (ERINLG-261) with antimicrobial and antiproliferative properties. Rev. Bras. Farmacogn., v.26, p.285-295, 2016.

BARBOSA, R.G.; BORGHESAN, A.C.; CERQUEIRA, N.F. et al. Fisiopatologia da trombose da veia jugular em equinos: revisão. Vet. Zootec. v.16, p.26-37, 2009 .

BHAVANI, B.; NAVEENA, B.; PARTHA, N. Strain improvement of Streptomyces venezuelae for enhanced fibrinolytic enzyme production. Adv. Mater. Res., v.584, p.440-444, 2012.

CASTRO, H.C.; ABREU, P.A.; GERALDO, R.B. et $a l$. Looking at the proteases from a simple perspective. J. Mol. Recognit., v.24, p.165-181, 2011

CHOI, D.; CHA, W.; PARK, N. et al. Purification and characterization of a novel fibrinolytic enzyme from fruiting bodies of Korean Cordycepsmilitaris. Bioresor. Technol., v.102, p.3279-3285, 2011.

CICHA, I. Thrombosis: novel nanomedical concepts of diagnosis and treatment. World J. Cardiol., v.7, p.434-441, 2015.

DEMIR, T.; HAMES, E.E.; SUPHI, S.O. et al. An optimization approach to scale up keratinase production by Streptomyces sp. $2 \mathrm{M} 21$ by utilizing chicken feather. Int. Biodeter. Biodegr., v.103, p.134140, 2015.

ESCODRO, P.B.; LOPES, P.F.R.; GIANINI, C.G. et al. Diagnóstico diferencial de trombose artoilíaca e mieloencefalite protozoária equina: relato de caso. Arq. Bras. Med. Vet. Zootec., v.62, p.1048-1053, 2010.

GINTHER.C. L. Sporulation and the production of serine protese and cephamycin C by Streptomyces lactamdurans. Antimicrob. Agents Chemother., v.15, p.522-526, 1979.

JU, X.; CAO, X.; SUN, Y. et al. Purification and characterization of a fibrinolytic enzyme from Streptomyces sp. XZNUM 00004. World J. Microbiol. Biotechnol., v.28, p.2479-2486, 2012.

MANDER, P.; CHO, S.S.; SIMKHADA, J.R. et al. A low molecular weight chymotrypsin-like novel fibrinolytic enzyme from Streptomycessp. CS624. Process Biochem., v.46, p.1449-1455, 2011.
NARAYANA, K.; VIJAYALAKSHMI, M. Optimization of antimicrobial metabolites production by Streptomyces albidoflavus. Res. J. Pharmacol.,v.2, p.4-7, 2008.

NASCIMENTO, T.P.; SALES, A.E.; PORTO, C.S. et al. Purification of a fibrinolytic protease from Mucorsubtilissimus UCP 1262 by aqueous two-phase systems (PEG/sulfate). J. Chromatogr. B., v.1025, p.16-24, 2016.

OYAMADA, T.; SAIGAMI, K.; PARK, C. et al. Pathology of aortic-iliac thrombosis in two horses. $J$. Equine Vet. Sci., v.18, p.59-65, 2007.

PORTO, A.L.F.; CAMPOS-TAKAKI, G.M.; LIMA FILHO, J.L. Effects of culture conditions on protease production by Streptomyces clavuligerus growing on the soy bean flour medium. Appl. Biochem. Biotechnol., v.60, p.115-122, 1996.

PRIDHAM, T.G.; ADERSON, P.; FOLEY, C. et al. Selection of media for maintenance and taxonomic study of Streptomyces. Antibiot. Ann., p.947-953, 1957.

SANCHEZ, S.; DEMAIN, A.L. Metabolic regulation of fermentation processes. Enzyme Microb. Technol.,v.31, p.895-906, 2002.

SILVA, G.M.M.; BEZERRA, R.P.; TEIXEIRA, J.A. et al. Fibrinolytic protease production by new Streptomyces sp. DPUA 1576. Electron. J. Biotechnol., v.18, p.16-19, 2015.

SILVA, G.M.M.; BEZERRA, R.P.; TEIXEIRA, J.A. et al. Screening, production and biochemical characterization of a new fibrinolytic enzyme produced by Streptomyces sp. (Streptomycetaceae) isolated from Amazonian lichens. Acta Amaz., v.46, p.301-310, 2016.

SIMKHADA, J.R.; MANDER, P.; CHO, S.S. et al. A novel fibrinolytic protease from Streptomyces sp. CS684. Process Biochem., v.45, p.88-93, 2010.

SOBREIRA, M.L.; YOSHIDA, W.M.; LASTÓRIA, S. Tromboflebite superficial: epidemiologia, fisiopatologia, diagnóstico e tratamento. J. Vasc. Bras., v.40, p.131-143, 2008.

STATISTICA for Windows: data analysis software system. Version 8.0. Tulsa: Statsoft., 2008. 298 p.

WANG, S.; WU, Y.; LIANG, T. Purification and biochemical characterization of a nattokinase by conversion of shrimp shell with Bacillus subtilis TKU007. N. Biotechnol., v.28, p.196-202, 2011. 